\title{
A Software-Defined Multifunctional Radar Sensor for Linear and Reciprocal Displacement Measurement
}

\author{
Li Lu${ }^{1}$, Changzhi $\mathrm{Li}^{1}$, and Jennifer A. Rice ${ }^{2}$ \\ ${ }^{1}$ Department of Electrical and Computer Engineering, Texas Tech University, Lubbock, Texas, 79409, USA \\ ${ }^{2}$ Department of Civil and Environmental Engineering, Texas Tech University, Lubbock, Texas, 79409, USA \\ Email: li.lu@ttu.edu,changzhi.li@ttu.edu,jennifer.rice@ttu.edu
}

\begin{abstract}
A software-defined multifunctional radar sensor is developed in this paper for linear and reciprocal displacement measurement. Experiments were performed to demonstrate the high accuracy of the two measurement methodologies. When configured in arctangent-demodulated interferometry mode with a $5.46 \mathrm{GHz}$ carrier frequency, the sensor can measure the displacement with sub-millimeter error at a detection distance of $1.2 \mathrm{~m}$. When configured in nonlinear vibrometer mode, the sensor can measure amplitudes of reciprocal motions with a resolution of 0.4 millimeter and less than $3 \%$ average error.
\end{abstract}

Index Terms - radar sensor, multifunctional, arctangentdemodulated interferometry, nonlinear vibrometer.

\section{INTRODUCTION}

Due to the advantages of wireless operation, the ability to be integrated into small semiconductor chips, and high detection accuracy, microwave and millimeter-wave radars have been used for displacement and motion sensing. Typical applications include noncontact vital sign detection [1], wireless local positioning [2], automotive driving assistance systems (ADAS) [3], etc. Taking advantage of RF/analog circuits' rapid scaling down of feature size and performance improvements, compact portable radar systems also find great potential in applications such as structural health monitoring (SHM) most recently. According to the American Society of Civil Engineers (ASCE), 'As of 2005, 156,335 of the nation's 595,363 bridges, or $26.3 \%$, were structurally deficient or functionally obsolete' [4]. Traditionally, accelerometers and global positioning system (GPS) are used for dynamic structural health monitoring. However, such devices have limited resolution and problems in detecting slow structural movements. Other displacement sensors, such as linear variable differential transformers (LVDTs), need to be placed in contact with the surveyed structure, which presents challenges in deployment for many structures. Radar technology has been recently used for structural health monitoring and overcomes the problems of using accelerometers, GPS, and LVDTs [5]. Another emerging application for radar technology is the noncontact pulse wave velocity (PWV) measurement in health care. Without anything attached to the human body, two pulses can be monitored by radar sensors. The transit time can be obtained from the demodulated phase difference of the two pulse waveforms and the PWV can be calculated based on the pulse travelling distance [6].

Although radar displacement/motion sensors are attractive for plenty of advantages, the lack of flexibility for a single unit to cover different applications is a problem associated with conventional radar. For example, a radar based on microwave interferometry has high detection accuracy in sub-millimeter range, but it has limited ability to detect slow movement [7][8]. Frequency modulated continuous wave (FMCW) radar measures absolute distance with high performance, but it is not efficient when detecting periodic movement such as vibration, since it needs to sample the target position at a frequency that is much higher than the fundamental vibration frequency to recover a complex movement pattern. A microwave vibrometer is recently invented with high accuracy when detecting vibration [9], but it does not directly monitor non-periodic movement.

This paper presents a software-defined multifunctional radar sensor that enables multiple detection modes for optimal measurement of both linear and reciprocal displacement. When configured in an arctangentdemodulated interferometery mode, the radar measures linear displacement (i.e. non-periodic movements) with high accuracy, since the displacement is resolved within a fraction of a wavelength of the operating frequency. When configured in the nonlinear vibrometer mode, the radar measures reciprocal movement (i.e. vibration) with large detectable movement range and sub-millimeter accuracy. Such a radar sensor will have large potential to advance wireless displacement/motion sensing in applications such as bridge monitoring and noncontact pulse wave velocity detection for health care applications.

This paper is organized as follows: Section II introduces the software-defined configuration for multiple mode operation. Experimental results are presented in section III. Section IV compares the two measurement methodologies. A conclusion is drawn in Section IV.

\section{THEORY}

Fig. 1 (a) shows the block diagram of the proposed software-defined radar. The radar LO can generate a 5-6 $\mathrm{GHz}$ continuous wave signal and is amplified and transmitted through the transmitting antenna. The reflected 
signal from the target is captured by the antenna and down-converted into baseband $I / Q$ signals by a quadrature mixer. The baseband signal is further amplified before it is digitized and fed into a digital signal processor (DSP) unit or a laptop for real time signal processing.

\section{A. Arctangent-demodulated interferometery mode}

For linear motion measurement, arctangent demodulation technique is adopted to reconstruct the phase information, which corresponds to the displacement of the target. DC calibration and mismatch correction are applied so that the reconstructed phase information is correct and accurate. The baseband $I / Q$ signals are described as

$$
\begin{aligned}
& B_{I}(t)=A_{I} \cos [\theta+4 \pi x(t) / \lambda+\Delta \varphi]+D C_{I} \\
& B_{Q}(t)=A_{Q} \sin [\theta+4 \pi x(t) / \lambda+\Delta \varphi]+D C_{Q}
\end{aligned}
$$

where $A_{\mathrm{I}}$ and $A_{\mathrm{Q}}$ are the amplitudes of the $I$ and $Q$ signals, $\theta$ is a constant phase shift due to the detection distance and the reflection at the surface of the target, $\Delta \varphi$ is the phase noise of the radar, and $\mathrm{DC}_{\mathrm{I}} / \mathrm{DC}_{\mathrm{Q}}$ are the $\mathrm{DC}$ offsets in $I / Q$ baseband signals, respectively. $4 \pi x(t) / \lambda$ is the phase information corresponding to the target movement. After the DC calibration and the mismatch correction, the $I / Q$ baseband signals will be expressed as

$$
\begin{aligned}
& B_{I}(t)=\cos [\theta+4 \pi x(t) / \lambda+\Delta \varphi] \\
& B_{Q}(t)=\sin [\theta+4 \pi x(t) / \lambda+\Delta \varphi]
\end{aligned}
$$

Accurate phase information can be obtained by arctangent demodulation as

$\psi(t)=\arctan \left[B_{Q}(t) / B_{I}(t)\right]+F=\theta+4 \pi x(t) / \lambda+\Delta \varphi+F$

where $F$ is a multiple of $180^{\circ}$ for the purpose of eliminating the discontinuity when $\psi(t)$ crosses the boundary of two adjacent quadrants in the constellation graph. The initial position of the target decides a specific $\theta . \varphi(t)=4 \pi x(t) / \lambda+F$ indicates the displacement of the subject being measured. The displacement of the target can be obtained as $x(t)=x_{0}+\varphi(t) \lambda / 4 \pi$, where $x_{0}$ is the initial position of the target.

\section{B. Nonlinear vibrometer mode}

The cosine or sine transfer function nonlinearly modulates the movement signal $x(t)$ as indicated in Eq. (1) and (2). Based on the measurement of the nonlinear effect inherent in the Doppler phase modulation mechanism, the same radar hardware can also precisely measure the amplitude of a reciprocal motion [9] when configured in a nonlinear vibrometer mode. Complex demodulation technique is adopted to construct the combined spectrum from the $I / Q$ basebands. By analyzing the relative strength (a)

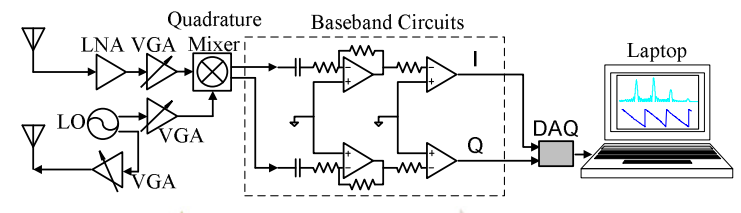

(b)

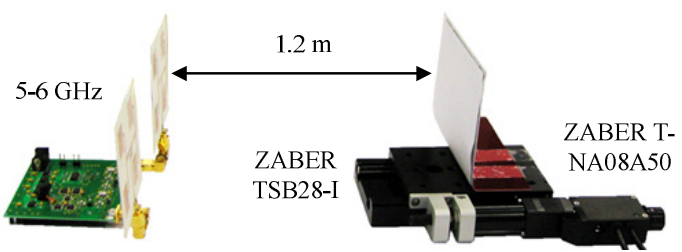

Fig. 1. (a) Block diagram of the radar sensor (b) Experiment setup

among the harmonics caused by nonlinear modulation, accurate amplitude of the reciprocal motion can be obtained.

Since a complex movement can be decomposed into a series of single tone movements, a single tone periodic movement, $x(t)=m \sin (\omega t)$, is studied here without lose of generality, where $\mathrm{m}$ and $\omega$ are the movement amplitude and frequency respectively. Let $\phi=\theta+\Delta \varphi$, which is the total residual phase. Eq. (3) and (4) can be combined as:

$$
\begin{aligned}
S(t) & =B_{I}(t)+j \cdot B_{Q}(t)=\exp \{j[4 \pi x(t) / \lambda+\phi]\} \\
& =2 \sum_{k=1}^{\infty} J_{k}(4 \pi m / \lambda) \cdot e^{j k \omega t} \cdot e^{j \phi}
\end{aligned}
$$

where $J_{n}(x)$ is the $\mathrm{n}^{\text {th }}$-order Bessel function of the first kind. Since $e^{j \phi}$ has a constant-envelope of unity, the relative strength among the frequency components (harmonics) is decided by the movement amplitude rather than affected by the receiver gain or the detecting distance, as presented in the following equation:

$$
H_{1}: H_{2}: H_{3}: \cdots=\left|J_{1}(r)\right|:\left|J_{2}(r)\right|:\left|J_{3}(r)\right|: \cdots
$$

Where $r=4 \pi m / \lambda$. The movement amplitude $m$ can be found by fitting the measured harmonic ratio to the theoretical value from Bessel function. The ratio of $\mathrm{H}_{2}$ and $H_{1}$ is adopted in this study in order to obtain maximum measurement resolution.

\section{EXPERIMENTAL RESULTS}

A 5- $6 \mathrm{GHz}$ quadrature radar sensor has been designed and fabricated on a print circuit board (PCB). As shown in Fig. 1 (a), a 5-6 GHz quadrature transceiver and a twostage baseband amplifier were integrated on a single $6.8 \times$ $7.5 \mathrm{~cm}^{2}$ PCB (Rogers RO4350B substrate). An NI USB6009 data acquisition module (DAQ) samples the I/Q baseband signals and feeds them into a laptop for real-time signal processing. A set of linear actuator (ZABER TNA08A50) and linear translation stage (ZABER TSB28-I) was placed $1.2 \mathrm{~m}$ in front of the radar antennas and was programmed to provide desired motion as shown in Fig. 1 
(a)

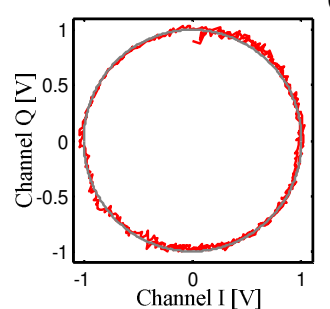

(b)

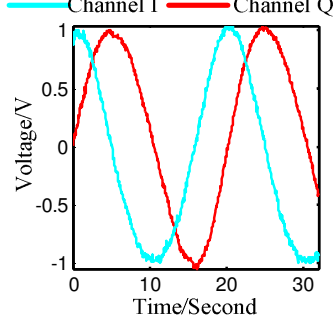

(c)

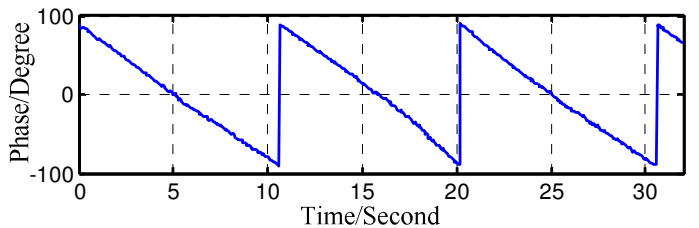

(d)

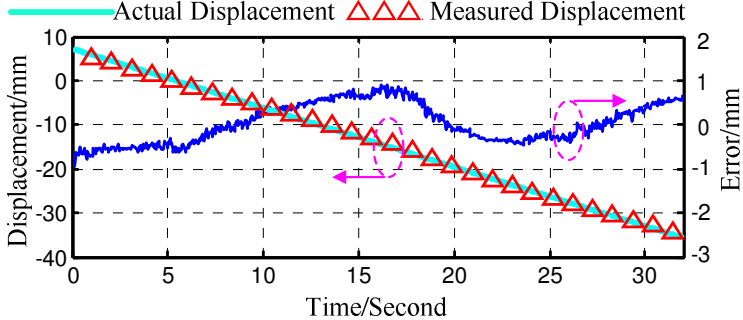

Fig. 2. (a) Constellation graph (b) Baseband signals after DC mismatch calibration (c) Phase information of arctangent demodulation (d) Measured displacement and the error.

(b). The actuator has a default resolution of $0.047625 \mu \mathrm{m}$, a high accuracy of $8 \mu \mathrm{m} / 50.8 \mathrm{~mm}$, a repeatability of less than $1 \mu \mathrm{m}$. For experiments in the arctangent-demodulated interferometry mode, a linear motion with a constant speed and a linear motion with two different speeds were generated by the linear actuator. It should be noted that these two movement patterns were adopted for the convenience of evaluating measurement error. The arctangent-demodulated interferometry mode can also measure more complicated movement styles such as an accelerated motion or general linear variable motions. For the verification of reciprocal movement measurement, a series of single tone sinusoidal motions with different movement amplitudes were generated by the linear actuator.

\section{A. Linear displacement measurement}

In the first measurement, the linear actuator was programmed to perform a linear motion with a speed of $1.33 \mathrm{~mm} / \mathrm{s}$. Before the arctangent demodulation, DC calibration and mismatch correction were performed with the help of constellation graph as shown in Fig. 2 (a) [6]. Fig. 2 (b) shows the I/Q baseband signals after the DC and mismatch calibration, which indicates the 90 degree phase shift between the two channels. The arctangentdemodulated phase information is presented in Fig. 2 (c). The phase is wrapped in the $-90^{\circ}$ to $90^{\circ}$ range. The phase correction factor $F$ (integer times of $180^{\circ}$ ) was applied in (a)

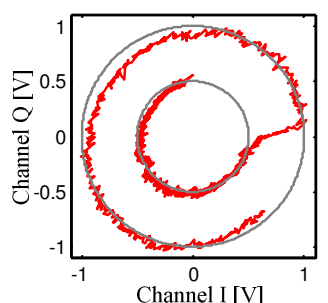

(b)

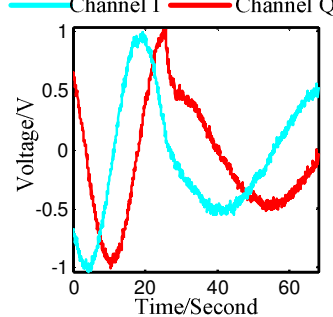

(c)

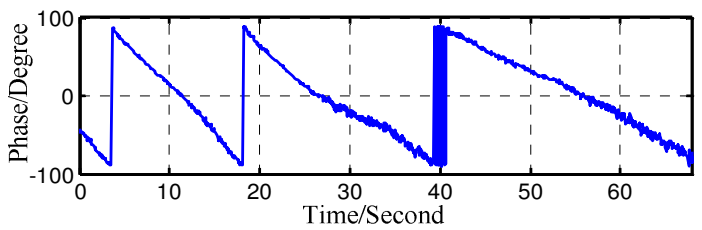

(d)

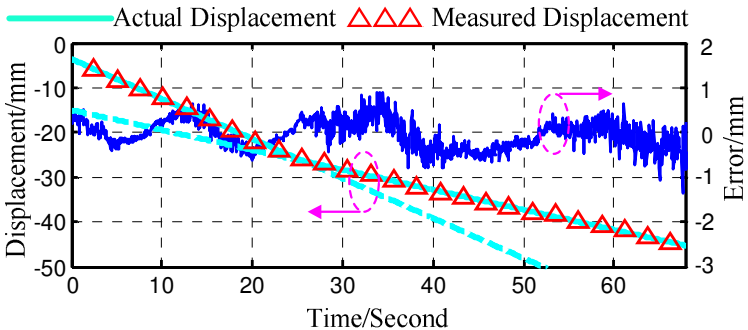

Fig. 3. (a) Constellation graph (b) Baseband signals after DC and mismatch calibration (c) Phase information of arctangent demodulation (d) Measured displacement and the error.

order to unwrap the measured phase. The unwrapped phase was further converted to the target displacement as shown in Fig. 2 (d). In addition, the programmed displacement of the target and the measured error are presented in Fig. 2 (d), where an error of less than $\pm 1 \mathrm{~mm}$ was observed.

Another motion with a speed jumped from $0.92 \mathrm{~mm} / \mathrm{s}$ to $0.46 \mathrm{~mm} / \mathrm{s}$ was programmed to further verify the high accuracy of the radar in arctangent-demodulated interferometry mode. Similar signal processing procedure was applied. Fig. 3 presents the measurement result. The radar successfully tracked the speed change and the maximum error is around $\pm 1 \mathrm{~mm}$.

The measurement error is mainly attributed to the nonlinear response of the quadrature mixer. Several techniques have been proposed to correct the nonlinearity of the quadrature mixer [10], which may further improve the accuracy of the measurement. The normalized error (normalized to the carrier wavelength $\lambda$ ) before error correction of this study is 0.018 , which is less than those in [7] (0.038) and [8] (0.033).

\section{B. Reciprocal amplitude measurement}

The linear actuator was programmed in a series of sinusoidal motions, which had the same $0.6 \mathrm{~Hz}$ frequency but had different amplitudes from $0.3 \mathrm{~mm}$ to $2 \mathrm{~mm}$ with a step size of $0.1 \mathrm{~mm}$. Complex demodulation was used to combine the two channels and construct the spectrum, 
(a)

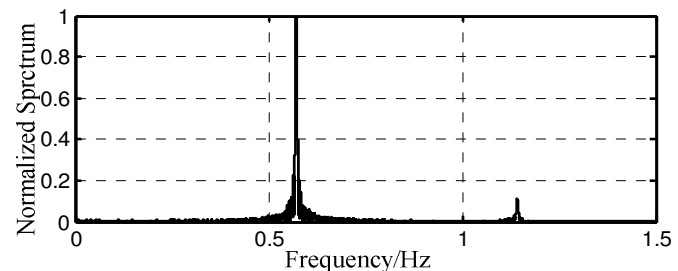

(b)

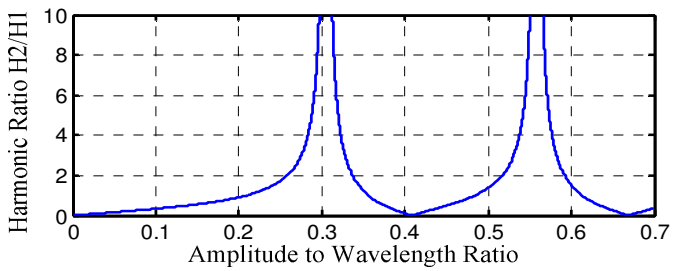

Fig. 4. (a) Baseband spectrum when detecting $2 \mathrm{~mm}$ sinusoidal movement; (b) Theoretic harmonic ratio $\mathrm{H} 2 / \mathrm{H} 1$

from which the $1^{\text {st }}$ and the $2^{\text {nd }}$ harmonics were identified. The ratio of the harmonics $(H 2 / H 1)$ was fitted into the theoretic value from Bessel function so that the amplitude of the movement was read out accordingly. Fig. 4 (a) shows the combined spectrum of the sinusoidal motion with $2 \mathrm{~mm}$ amplitude while Fig. 4 (b) plots the theoretic $H 2 / H 1$ vs. the ratio of the amplitude and the carrier wavelength. Table I presents the measurement results for amplitude $<1 \mathrm{~mm}$. The average measurement error is less than $3 \%$ of the motion amplitudes when the amplitudes are larger than $0.3 \mathrm{~mm}$. Therefore, the measurement resolution is around $0.4 \mathrm{~mm}$ at a $1.2 \mathrm{~m}$ detection distance. The measurement resolution can be further improved if the detection distance is reduced to improve the signal-tonoise ratio.

\section{COMPARISON OF MEASUREMENT MODES}

Table II summerized the performance of the two measurement mechanisms with a $5.46 \mathrm{GHz}$ carrier frequency and $1.2 \mathrm{~m}$ detection distance. Both of the two measurement mechanisms integrated in the proposed radar sensor platform have high measurement accuracy and can be applied in various areas as demonstrated in the experiments. The limitation of the arctangent-demoduated interferometry mode is that only relative displacement can be measured without the information of the absolute distance from a target while the disadvantage of the nonlinear vibrometer mode is that it can be only applied to reciprocal motions.

\section{CONCLUSION}

The proposed radar sensor is featured by softwareconfigured multiple functions with high measurement accuracy. Experiments were performed to demonstrate the feasibility of the multiple measurement mechanisms. At $5.46 \mathrm{GHz}$ carrier frequency and $1.2 \mathrm{~m}$ detection distance, the arctangent-demodulated interferometry tracks the
TABLE I. PERFORMANCE OF NONLINEAR VIBROMETER

\begin{tabular}{|c|c|c|c|c|}
\hline $\begin{array}{c}\text { Amplitude } \\
(\mathbf{m m})\end{array}$ & $\begin{array}{c}\text { Worst } \\
(\mathbf{m m})\end{array}$ & $\begin{array}{c}\text { Best } \\
(\mathbf{m m})\end{array}$ & $\begin{array}{c}\text { Average } \\
(\mathbf{m m})\end{array}$ & $\begin{array}{c}\text { Average } \\
\text { error }\end{array}$ \\
\hline 0.3 & 0.410 & 0.338 & 0.335 & $11.7 \%$ \\
\hline 0.4 & 0.356 & 0.402 & 0.406 & $1.6 \%$ \\
\hline 0.5 & 0.524 & 0.485 & 0.511 & $2.2 \%$ \\
\hline 1 & 1.026 & 0.999 & 1.006 & $0.6 \%$ \\
\hline
\end{tabular}

TABLE II. COMPARISON OF THE MEASUREMENT MODES

\begin{tabular}{|c|c|c|}
\hline $\begin{array}{c}\text { Measurement } \\
\text { Mode }\end{array}$ & $\begin{array}{c}\text { Arctangent-Demodulated } \\
\text { Interferometry }\end{array}$ & $\begin{array}{c}\text { Nonlinear } \\
\text { Vibrometer }\end{array}$ \\
\hline Motion Pattern & Linear & Reciprocal \\
\hline Resolution & ---- & $0.4 \mathrm{~mm}$ \\
\hline $\begin{array}{c}\text { Measurement } \\
\text { Error }\end{array}$ & $<1 \mathrm{~mm}$ & $\begin{array}{c}<0.13 \mathrm{~mm} \text { worst } \\
<0.03 \mathrm{~mm} \text { aver. }\end{array}$ \\
\hline Limitation & $\begin{array}{c}\text { Only measures relative } \\
\text { displacement }\end{array}$ & $\begin{array}{c}\text { Only measures } \\
\text { reciprocal motion }\end{array}$ \\
\hline
\end{tabular}

target position with less than $1 \mathrm{~mm}$ error, while with the nonlinear methodology the radar precisely measures amplitudes of reciprocal motions with $0.4 \mathrm{~mm}$ resolution and less than $3 \%$ average error.

\section{REFERENCES}

[1] C. Li, J. Cummings, J. Lam, E. Graves, W. Wu, "Radar remote monitoring of vital signs," IEEE Microwave Magazine, vol. 10, issue 1, pp. 47-56, February 2009.

[2] M. Vossiek, L. Wiebking, P. Gulden, J. Wieghardt, C. Hoffmann, P. Heide, "Wireless local positioning," IEEE Microwave Magazine, vol. 4, issue 4, pp. 77-86, 2003.

[3] M. Russel, A. Crain, R. Campbell, A. Clifford, W. Miccioli, "Millimetre wave radar sensor for automotive intelligent cruise control," IEEE Trans. Microwave Theory Techn. vol. 45, pp. 2444-2452, 1997.

[4] The American Society of Civil Engineers (ASCE): http://w ww.asce.org/pressroom/news/policy_details.cfm?hdlid=132

[5] M. Pieraccini, M. Fratini, F. Parrini, C. Atzeni, "Dynamic monitoring of bridges using a high-speed coherent radar," IEEE Trans. Geoscience and remote sensing, vol. 11, pp. 3284-3288, November 2006.

[6] L. Lu, C. Li and D. Lie, "Experimental demonstration of noncontact pulse wave velocity monitoring using multiple doppler radar sensors," 32nd Annual International Conference of the IEEE Engineering in Medicine and Biology Society, Argentina, September 2010.

[7] S. Kim and N. Cam, "A displacement measurement technique using millimeter-wave interferometry," IEEE Trans. Microwave Theory and Techniques, vol. 51, pp. 1724-1728, 2003.

[8] S. Kim and N. Cam, "On the development of a multifunction millimeter-wave sensor for displacement sensing and low-velocity measurement," IEEE Trans. Microwave Theory and Tech., vol. 52, pp. 2503-2512, 2004.

[9] C. Li and J. Lin. "Non-contact measurement of periodic movement by a $22-40 \mathrm{GHz}$ radar sensor using nonlinear phase modulation," IEEE MTT-S Int. Microwave Symp. Dig., pp. 579-582, Honolulu, June 2007.

[10]D. E. Noon, "Wide band quadrature error correction (using SVD) for stepped-frequency radar receivers," IEEE Trans. Aerosp. Electron. Syst., vol. 35, pp. 1444-1449, Oct. 1999. 